\title{
Effective treatment of low-dose decitabine in myelodysplastic syndrome/myeloproliferative neoplasms
}

\author{
Xingnong Ye \\ Dan Chen \\ Yan Zheng \\ Xiaoqiong Zhu \\ Junkai Fu \\ Jian Huang
}

Department of Hematology, The Fourth Affiliated Hospital, College of Medicine, Zhejiang University,

Zhejiang Province, People's

Republic of China

\author{
This article was published in the following Dove Press journal: \\ OncoTargets and Therapy \\ 13 November 2017 \\ Number of times this article has been viewed
}

Objective: Primary myelofibrosis (PMF) is one of the Philadelphia negative myeloproliferative neoplasms (MPN). The main clinical features are obvious physical symptoms and symptomatic splenomegaly. It may be converse to leukemia and has a shortened life expectancy. Nowadays, the therapy for PMF is aimed at maintaining comfort and there is no curative treatment. PMF with myelodysplastic syndrome (MDS), called MDS/MPN-u, is rare and the treatment is complex. In this study, we want to discuss an effective treatment for MDS/MPN via a case report and literature review.

Materials and methods: A female patient was diagnosed with MDS/MPN through bone marrow cytology, immunology, cell genetics, molecular biology, and pathology. She received thalidomide and prednisone as initial treatment. Ten months later, the first-line therapy had failed, she presented with clinically relevant pancytopenia and increased blasts in bone marrow. Because decitabine is one of the first-line treatments for MDS and the patient was frail, she received low-dose decitabine as second-line therapy. Decitabine was administered at $15 \mathrm{mg} / \mathrm{m}^{2}$ once a week for 3 weeks, in a 4 week cycle. If there was improvement the treatment interval was prolonged.

Result: After one cycle, the blasts in bone marrow were decreased to $0.5 \%$. After four cycles, she felt comfortable and hematological improvement was achieved. The treatment interval was prolonged. After eight cycles, the spleen reduced to $2 \mathrm{~cm}$ under the rib, and she achieved complete hematological remission. After ten cycles, the mutation of JAK2/V617F expression was decreased from $60.63 \%$ to $0.01 \%$. During the therapy, the patient presented with grade III-IV hematological toxicity after the first two cycles, but there were no side effects after subsequent cycles.

Conclusion: Our research showed that low-dose decitabine may be an effective treatment for MDS/MPN, especially in improving physical symptoms and achieving hematological remission. Besides, it may be possible to reverse positive JAK2 mutation.

Keywords: decitabine, myelodysplastic syndrome, myeloproliferative neoplasms

\section{Introduction}

Myelodysplastic syndrome (MDS)/myeloproliferative neoplasms (MPN) (MDS/MPN) is a set of clonal malignant disorders of hematopoietic stem cells. These diseases are characterized as having some clinical/laboratory or morphological features of MDS, and some other manifestations of chronic MPN. Primary marrow fibrosis (PMF) is one of the Philadelphia negative MPN, which is characterized by clonal proliferation of megakaryocytes and fibrous tissue in the bone marrow (BM). The clinical manifestations of PMF include: shortened life expectancy, endothelial dysfunction,
Department of Hematology, The Fourth Affiliated Hospital, College of Medicine, Zhejiang University, NI Shangchengdadao Road, Yiwu 322000, Zhejiang Province,

People's Republic of China

Tel +86 I88 6796 I032

Fax +86 57989935555

Email househuangjian@।63.com

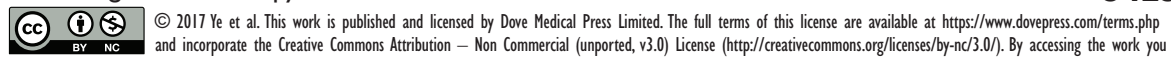
BY NC and incorporate the Creative Commons Attribution - Non Commercial (unported, v3.0) License (http://creativecommons.org/licenses/by-nc/3.0/). By accessing the work you
hereby accept the Terms. Non-commercial uses of the work are permitted without any further permission from Dove Medical Press Limited, provided the work is properly attributed. For permission for commercial use of this work, please see paragraphs 4.2 and 5 of our Terms (https://www.dovepress.com/terms.php).
forition 
vascular events, significant hepatosplenomegaly, obvious anorexia, cachexia, and possible transformation to acute leukemia. ${ }^{1}$ MDS is a heterogeneous group of disorders characterized by impaired peripheral blood cell production (cytopenias) and most commonly hypercellular, dysplasticappearing BM. ${ }^{2,3}$ Patients with intermediate/high-risk MDS (International Prognostic Scoring System grouping) typically progress to acute myeloid leukemia (AML) when in advanced stage, for which treatment options are limited and outcomes are generally poor. ${ }^{4} \mathrm{PMF}$ complicated by MDS, called MDS/ MPN- $\mathrm{u}$, is rare. Therapy is aimed at maintaining comfort and there is no curative treatment.

Previous studies have demonstrated that abnormal methylation patterns characterize a significant subset of patients with myeloid malignancies. ${ }^{5,6} 5$-aza-2'-deoxycytidine (DAC) is a DNA methyltransferase (DNMT) inhibitor which reactivates aberrantly silenced genes through the reversal of abnormal DNA methylation. DAC was approved by the United States Food and Drug Administration (FDA) for the treatment of MDS in 2006 and the European Medicine Agency for the treatment of AML in elderly patients (over 65 years of age) in 2012. In clinical, DAC was originally used to treat patients with intermediate and high-risk MDS and was then identified for use in elderly patients with AML and relapsed/ refractory AML. However, there is a lack of research regarding treating MDS/MPN with DAC, and the effect has been confusing. The FDA-approved DAC regimens to treat MDS were $20-45 \mathrm{mg} / \mathrm{m}^{2} /$ day for 3 to 5 days every 4 to 6 weeks, which can have cytotoxic effects. Reducing DAC dosages may alleviate or avoid cytotoxicity. Here we report a case of MDS/MPN, which was successfully treated with low-dose DAC after initial therapy failure. It showed that low-dose DAC may be an effective treatment for MDS/MPN.

\section{Materials and methods}

A 55-year-old woman presented with fatigue and chest distress for 1 month and was admitted to our hospital. Physical examinations showed anemic appearance and splenomegaly (four fingers under the rib). A routine blood test showed an HB level of $84 \mathrm{~g} / \mathrm{L}$, the white blood cell (WBC) count was $1,200 / \mu \mathrm{L}$, while the platelet blood count (PBC) was $82,000 / \mu \mathrm{L}$, and blood smear showed tear drop-shaped cells. BM cytology showed that blasts accounted for 3\%, accompanied by increased erythrocytes. BM pathology showed active hyperplasia and megakaryocyte proliferation and atypia and fibrous tissue proliferation. The JAK2 mutation was positive and the expression of JAK2/V617F was $60.63 \%$. The CSF3R, KRAS, and NRAS mutations were all negative.
The chromosome karyotype showed 47, XX, t $(1 ; 20)$ (p11.2; q11.2), +9, -13, +21. She was diagnosed with MDS/MPN-u according to the World Health Organization's 2016 version of hematologic neoplasms classification, which was PMF complicated with MDS (Table 1). In addition, she had a history of ankylosing spondylitis and interstitial pneumonia, and she was on long-term therapy of prednisone $10 \mathrm{mg}$ daily. After MDS/MPN-u was diagnosed, she received thalidomide $100 \mathrm{mg}$ daily combined with prednisone. As a result her fatigue improved and blood examination was stable. The WBC count was 1,800-2,800/ $\mu \mathrm{L}, \mathrm{HB}$ level was 82-105 g/L, and $\mathrm{PBC}$ was $70,000-90,000 / \mu \mathrm{L}$. After 6 months' treatment, she underwent repeated BM examination which showed that the disease was stable, and there were less than 5\% blasts.

Ten months later, she felt uncomfortable and progressive fatigue, accompanied by abdominal distention. Besides, a physical exam revealed aggravated splenomegaly (one palm under navel and right edge over midline). She then underwent blood and $\mathrm{BM}$ examination again. A routine blood test showed WBC count was $1,600 / \mu \mathrm{L}$, HB level was $80 \mathrm{~g} / \mathrm{L}$, and $\mathrm{PBC}$ was $62,000 / \mu \mathrm{L}$. Blood biochemistry showed that lactate dehydrogenase was $379 \mathrm{U} / \mathrm{L}, \beta 2$-macroglobulin was $5.68 \mathrm{mg} / \mathrm{L}$, and uric acid was $379 \mathrm{U} / \mathrm{L}$. BM cytology showed $8.5 \%$ blasts, and flow cytometry of BM showed that the myeloid progenitor cell accounted for $3.48 \%$ and the abnormal granulocyte accounted for $63.22 \%$ of the non-erythroid cells. The BM pathology showed extremely active hyperplasia, accompanied with collagen fibrosis grade 3 .

The patient presented with obvious physical symptoms and progressive splenomegaly, accompanied with increased blasts. These manifestations reflected that the disease was

Table I Patient characteristics for diagnosis

\section{Patient's characteristics}

Presence of megakaryocyte proliferation and atypia, accompanied by collagen fibrosis grade 3

Not meeting World Health Organization criteria for ET, PV, BCR/ABL positive CML, myelodysplastic syndromes, or other myeloid neoplasms Presence of JAK2 mutation

Palpable splenomegaly, confirmed during two consecutive examinations LDH increased to above upper normal limit of institutional reference range, confirmed during two consecutive determinations

Dysplasia and hypocytosis

Abnormal chromosome karyotype, no MDS defining chromosomal abnormality

$5 \%$ 20\% blasts in bone marrow cytology, no preceding history of MPN or MDS, no recent cytotoxic or growth factor therapy

Abbreviations: $\mathrm{ET}$, essential thrombocytosis; $\mathrm{PV}$, polycythemia vera; $\mathrm{CML}$, chronic myeloid leukemia; MDS, myelodysplastic syndrome; MPN, myeloproliferative neoplasms. 
progressing and the previous treatment (thalidomide and prednisone) had failed. She then received new therapy with low-dose DAC. She received DAC at $15 \mathrm{mg} / \mathrm{m}^{2}$ weekly via intravenous infusion over 1 hour. Once a week for 3 weeks, in a 4 week cycle. If the disease improved, the dosing interval would be extended.

\section{Ethics statement}

This study has been approved by the Ethics Committee of the Fourth Affiliated Hospital of Zhejiang University. Before collecting clinical isolates from the patient, we informed her of our research purposes and written informed consent was obtained. We advised that her specimens were for scientific purposes only, and the patient and specimens information were anonymous in order to protect the health, safety and privacy of the patient.

\section{Results and safety}

After two cycles, the BM cytology showed the blasts had decreased to $0.5 \%$. After four cycles, she felt comfortable and physical symptoms had disappeared. The routine blood monitoring showed WBC count was above $2,000 / \mu \mathrm{L}, \mathrm{HB}$ level was over $120 \mathrm{~g} / \mathrm{L}$, and PBC was above $75,000 / \mu \mathrm{L}$, thus, hematological improvement had been achieved. Besides, the spleen had shrunk. These results showed that the therapy was effective. From the fifth cycle, the dosing interval was extended to one dose every 10 days, and a course every 6 weeks. After six cycles, BM examination was repeated. The BM cytology showed $0.5 \%$ blasts and normal chromosome karyotype, while the expression of JAK2/V617F was decreased to $19.2 \%$. After eight cycles, she felt well and physical exam revealed splenomegaly of only 1 finger under the rib. After ten cycles, the expression of JAK2/V617F was decreased to $0.01 \%$.

The safety was assessed based on Common Terminology Criteria for Adverse Events version 4.0. During the therapy, the patient presented with grade III-IV hematological toxicity, including neutropenia, anemia, and thrombocytopenia, lasting 2 to 3 weeks, due to which the third dosing was suspended. Thus, for the first two cycles, the patient only received two doses of DAC. During the first cycle, drugrelated chest distress and pulmonary infection also occurred, but both were mild. During the BM suppression, the patient received G-CSF therapy and blood transfusion of red blood cells and platelets. During the subsequent eight cycles, no hematological toxicity occurred, and all the doses were administered. No other non-hematological toxicity was observed during treatments.

\section{Discussion}

PMF patients face a series of clinical problems, such as anemia, spleen enlargement, physical symptoms, and symptomatic medullary hematopoiesis. ${ }^{7-9}$ We should recognize and deal with these problems as early as possible and provide appropriate treatment. Nowadays, the treatment strategies are aimed at alleviating clinical problems, hematopoietic stem cell transplantation and JAK inhibitors, and so on. However, all these treatments merely delay disease progression and cannot eliminate the disease. Recently, various clinical studies have been conducted, but none achieved the desired results. Thalidomide is an immunomodulatory drug, and it may also be a cytokine modulator. It has been increasingly used in low-risk MDS and PMF in recent years. There have been more small studies in PMF with low-dose thalidomide therapy. In a confirmed Phase II clinical trial in PMF, thalidomide achieved good results, but with obvious side effects. After combining with prednisone, the side effects were significantly decreased, and the therapeutic effect was improved. ${ }^{10,11}$ Therefore, thalidomide combined with prednisone is used for PMF patients with moderate-tosevere anemia, and it is one of the first-line treatments for PMF at present.

At first this patient was diagnosed with MDS/MPN-u according to morphology, immunology, cytogenetics and molecular biology, with moderate anemia. She was given thalidomide and prednisone as first-line therapy. However, after 10-month therapy with thalidomide and prednisone, the disease had progressed. She presented with obvious physical symptoms, progressive splenomegaly, and gradually developed pancytopenia. Thus, she underwent BM examination again, which showed that the blasts had increased. This indicated that the disease might have progressed to acute leukemia. What is the second-line therapy?

Recently published studies have demonstrated that gene inactivation of tumor-suppressors due to abnormal DNA methylation partly contributes to the pathogenesis of PMF and MDS/MPN. ${ }^{12}$ DAC has been suggested to exert antineoplastic effects through the inhibition of DNMT, causing DNA hypomethylation, and is widely used in MDS and AML nowadays. DAC has been used in a Phase II clinical trial in 34 PMF patients. ${ }^{13}$ The results showed eight patients achieved clinical response, including one case of partial remission and seven cases of clinical improvement. However, it could not reverse the fibrous hyperplasia and JAK2 mutation. On the other hand, $43 \%$ of patients achieved a response in 76 chronic myelomonocytic leukemia patients treated with azacitidine (another inhibitor of DNMT), which also indicated that the 
inhibitors of DNMT were widely used in MDS/MPN. ${ }^{14}$ $\mathrm{DAC}$ also achieved clinical response in a clinical trial with 21 PMF patients. ${ }^{15}$

Previously mentioned research suggests that inhibitors of DNMT might play a role in MDS/MPN, especially PMF complicated with MDS. According to reports, low doses of DAC have been shown to induce epigenetic modulation, while high doses have cytotoxic effects. ${ }^{16}$ Saunthararajah et al reported a clinical trial with low-dose DAC in MDS. ${ }^{17}$ Twenty-five patients with MDS received reduced DAC dosages $(0.1-0.2 \mathrm{mg} / \mathrm{kg} /$ day compared with the FDAapproved $20-45 \mathrm{mg} / \mathrm{m}^{2} /$ day dosage, a $75 \%-90 \%$ reduction), administered 1-3 days per week, instead of pulse cycled for 3-5 days over a 4-6-week period. The results showed that DAC regimens can be redesigned to minimize cytotoxicity and increase exposure time for DNMT1 depletion, to safely and effectively circumvent mutation apoptotic defects. In this patient, the therapeutic targets were aimed at alleviating the patient's symptoms, not a cure, and she was frail, so we adopted low-dose DAC treatment. After several cycles, the patient felt comfortable with the splenomegaly and pancytopenia approved significantly. The JAK2/V617F expression was decreased obviously. However, here was no change in fibrous proliferation. Besides, low-dose DAC showed good safety in this patient.

Our research indicated that low-dose DAC was an effective and safe treatment for MDS/MPN, especially in improving physical symptoms and pancytopenia, and it may be possible to reverse positive JAK2 mutation.

\section{Acknowledgment}

The research was supported by funding of the Science and Technology Department of Zhejiang Province, China (2016C33160), and Yiwu public technology research projects, Zhejiang Province, China (2016-S-05).

\section{Disclosure}

The authors report no conflicts of interest in this work.

\section{References}

1. Tefferi A, Thiele J, Orazi A, et al. Proposals and rationale for revision of the World Health Organization diagnostic criteria for polycythemia vera, essential thrombocythemia, and primary myelofibrosis: recommendations from an ad hoc international expert panel. Blood. 2007; 110(4):1092-1097.

2. Nimer SD. Myelodysplastic syndrome. Blood. 2008;111(10): $4841-4851$.

3. Steensma DP, Tefferi A. The myelodysplastic syndrome(s): a perspective and review highlighting current controversies. Leuk Res. 2003; 27(2):95-120

4. Van Etten RA, Shannon KM. Focus on myeloproliferative diseases and myelodysplastic syndromes. Cancer Cell. 2004;6(6):547-552.

5. Issa JP, Baylin SB, Herman JG. DNA methylation changes in hematologic malignancies: biologic and clinical implications. Leukemia. 1997;11 (Suppl 1):S7-S11.

6. Melki JR, Clark SJ. DNA methylation changes in leukaemia. Semin Cancer Biol. 2002;12(5):347-357.

7. Barosi G, Ambrosetti A, Finelli C, et al. The Italian Consensus Conference on Diagnostic Criteria for Myelofibrosis with Myeloid Metaplasia. Br J Haematol. 1999;104(4):730-737.

8. Barbui T, Barosi G, Birgegard G, et al. Philadelphia-negative classical myeloproliferative neoplasms: critical concepts and management recommendations from European LeukemiaNet. J Clin Oncol. 2011; 29(6):761-770.

9. Reilly JT, McMullin MF, Beer PA, et al. Guideline for the diagnosis and management of myelofibrosis. Br J Haematol. 2012;158(4):453-471.

10. Thomas DA, Giles FJ, Albitar M, et al. Thalidomide therapy for myelofibrosis with myeloid metaplasia. Cancer. 2006;106(9):1974-1984.

11. Mesa RA, Steensma DP, Pardanani A, et al. A phase 2 trial of combination low-dose thalidomide and prednisone for the treatment of myelofibrosis with myeloid metaplasia. Blood. 2003;101(7):2534-2541.

12. Nischal S, Bhattacharyya S, Christopeit M, et al. Methylome profiling reveals distinct alterations in phenotypic and mutational subgroups of myeloproliferative neoplasms. Cancer Res. 2013;73(3):1076-1085.

13. Quintas-Cardama A, Tong W, Kantarjian H, et al. A phase II study of 5-azacitidine for patients with primary and post-essential thrombocythemia/polycythemia vera myelofibrosis. Leukemia. 2008;22(5): 965-970.

14. Adès L, Sekeres MA, Wolfromm A, et al. Predictive factors of response and survival among chronic myelomonocytic leukemia patients treated with azacitidine. Leuk Res. 2013;37(6):609-613.

15. Odenike OM, Godwin JE, Van Besien K, et al. Phase II trial of low dose, subcutaneous decitabine in myelofibrosis. Poster presented at: 50th ASH Annual Meeting and Exposition; December 6-9, 2008; San Francisco, CA.

16. Qin T, Youssef EM, Jelinek J, Chen R, Yang AS, Garcia-Manero G, Issa JP. Effect of cytarabine and decitabine in combination in human leukemic cell lines. Clin Cancer Res. 2007;13(14):4225-4232.

17. Saunthararajah Y, Sekeres M, Advani A, et al. Evaluation of noncytotoxic DNMT1-depleting therapy in patients with myelodysplastic syndromes. J Clin Invest. 2015;125(3):1043-1055.
OncoTargets and Therapy

\section{Publish your work in this journal}

OncoTargets and Therapy is an international, peer-reviewed, open access journal focusing on the pathological basis of all cancers, potential targets for therapy and treatment protocols employed to improve the management of cancer patients. The journal also focuses on the impact of management programs and new therapeutic agents and protocols on

\section{Dovepress}

patient perspectives such as quality of life, adherence and satisfaction. The manuscript management system is completely online and includes a very quick and fair peer-review system, which is all easy to use. Visit http://www.dovepress.com/testimonials.php to read real quotes from published authors. 